\title{
Optic nerve sheath diameter measurement: a means of detecting increased intracranial pressure in pseudotumor cerebri patients
}

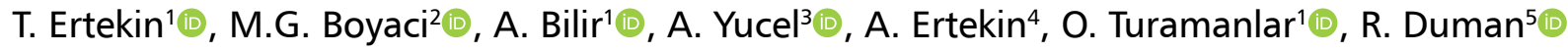 \\ ${ }^{1}$ Department of Anatomy, School of Medicine, Afyonkarahisar Health Sciences University, Afyonkarahisar, Turkey \\ 2Department of Neurosurgery, School of Medicine, Afyonkarahisar Health Sciences University, Afyonkarahisar, Turkey \\ ${ }^{3}$ Department of Radiology, School of Medicine, Afyonkarahisar Health Sciences University, Afyonkarahisar, Turkey \\ ${ }^{4}$ Department of Emergency Medicine, School of Medicine, Afyonkarahisar Health Sciences University, Afyonkarahisar, Turkey \\ ${ }^{5}$ Department of Opthalmology, Bursa Yüksek Ihtisas Training and Research Hospital, Bursa, Turkey
}

[Received: 20 August 2021; Accepted: 24 September 2021; Early publication date: 21 October 2021]

Background: Pseudotumor cerebri (PTC) occurs when the pressure inside the skull increases for no obvious reason. The aim of this study was to investigate three different methods: the optic nerve sheath diameter (ONSD) method, ONSD/eyeball transverse diameter (ETD) index, and ONSD/orbital transverse diameter (OTD) index for the initial detection of elevated intracranial pressure in patients with PTC. Materials and methods: A retrospective study of magnetic resonance data from adult PTC patients $(n=42)$ and control group $(n=40)$ was performed. ONSD and OTD measurements were made $3 \mathrm{~mm}$ and $10 \mathrm{~mm}$ posterior to the globe, after intracranial pressure was measured with lumbar puncture. The sensitivity, specificity, and overall accuracy of the findings on magnetic resonance imaging were calculated.

Results: The optic nerve sheath was enlarged in the PTC group compared with the control group. It showed $97 \%$ sensitivity and $100 \%$ specificity and $79 \%$ sensitivity and $87.5 \%$ specificity for $3 \mathrm{~mm}$ and $10 \mathrm{~mm}$, respectively. The ONSD/ IETD and ONSD/OTD indices were increased in the PTC group compared with the control group. For $3 \mathrm{~mm}$ posterior to the globe, the ONSD/ETD index had $90.5 \%$ sensitivity and 92\% specificity, and the ONSD/OTD index had $86 \%$ sensitivity and $95 \%$ specificity. For $10 \mathrm{~mm}$ posterior to the globe, the sensitivity and specificity of the ONSD/ETD and ONSD/OTD indices were $86 \%$ and $80 \%$ and $74 \%$ and $82.5 \%$, respectively.

Conclusions: According to our study, the ONSD method and the ONSD/ETD and ONSD/OTD indices are reliable diagnostic markers for PTC. These noninvasive techniques may be useful in monitoring the invasive intracranial catheter and have wide potential clinical applications in district hospitals, emergency departments and intensive care units. (Folia Morphol 2022; 81, 3: 567-573)

Key words: intracranial pressure, neurosurgery, optic nerve, pseudotumor cerebri, radiology

Address for correspondence: Dr. A. Bilir, Afyonkarahisar Health Sciences University, School of Medicine, Department of Anatomy, Afyonkarahisar, Turkey, tel: +90272 24628 42, fax: +90272 22814 29, e-mail: fztabdulkadirbilir@gmail.com

This article is available in open access under Creative Common Attribution-Non-Commercial-No Derivatives 4.0 International (CC BY-NC-ND 4.0) license, allowing to download articles and share them with others as long as they credit the authors and the publisher, but without permission to change them in any way or use them commercially. 


\section{INTRODUCTION}

Pseudotumor cerebri (PTC), also known as idiopathic intracranial hypertension, is a disorder that is described by the presence of pathologically increased intracranial pressure (ICP) in nonexistence of enlarged ventricles, intracranial mass lesions, and computed tomography (CT) and magnetic resonance (MR) imaging (MRI) findings associated with high ICP [1, 20, 25].

Intracranial pressure is detected by invasive placement into the ventricles or cerebral parenchyma with an intracranial catheter. This procedure is considered the gold standard. This method gives ICP results expressed in millimetres of mercury. Doctors tend to use noninvasive methods before applying invasive methods, and noninvasive methods are predominantly qualitative (e.g., papilledema, optic nerve sheath diameter [ONSD], tympanic membrane displacement) or provide imprecise readings $[2,18,19,21]$.

In this measurement method, the optic nerve sheath dilates due to more cerebrospinal fluid entering between the optic nerve and the dura mater. This is considered an indication of increased intracranial pressure [13, 14, 27].

Researchers have been mostly satisfied with the ONSD method and determined the correlation with the ICP measured by the invasive method, but some uncertainties and inconsistencies have remained regarding the accuracy of the method. Although multiple protocols and thresholds are suggested for the ONSD method, there is no generally accepted protocol or standardization. While ICP values greater than $20 \mathrm{mmHg}$ are considered pathological, no quantitative link has been established between ICP and ONSD beyond this value. But different researchers determined a normal/abnormal cutoff value of ONSD that varied from 4.8 to $7.3 \mathrm{~mm}[4,9,15,17,30]$.

Another thought-provoking point with the ONSD method is that ONSD measurements of normal and sick individuals in the same studies often have large standard deviations (SD) overlapping each other. To overcome this situation, researchers have proposed and attempted to apply different indices on various imaging techniques, such as the ratio of optic nerve diameter to ONSD or the ratio of ONSD to eyeball transverse diameter (ETD) $[6,27]$. The clinical application of the ONSD/ETD index has been described for patients with traumatic brain injury [28].

Our aim was to implement three different methods (the ONSD method, ONSD/ETD index, ONSD/ /orbital transverse diameter [OTD] index) for the initial detection of elevated ICP in patients with PTC. We investigated the applicability and reliability of the three methods in PTC patients.

\section{MATERIALS AND METHODS}

Study design and setting, inclusion and exclusion criteria

In this study, we retrospectively analysed the MR scan data of 82 adult patients (over 18 years old) who were admitted to the Radiology Department from January 2014 to December 2016. The present study was approved by the ethical committee of Afyonkarahisar Health Science University, Turkey (2017/7-193). In our study, we included patients who presented to the Neurosurgery Department in various combinations with complaints of headache, nausea, vomiting, visual impairment, diplopia, and dizziness and were directed to MR scans of the cranial region. The patients appeared to have negative MRI scans.

\section{Control group}

The control group was composed of patients older than 18 years who underwent MRI for various reasons (e.g., seizures, head injury, headache, and hearing loss), and the clinical results of these patients were evaluated as normal by a neuroradiologist. Patients whose signs and symptoms showed increased ICP were excluded from the control group. In addition, patients with a history of intracranial neoplasm, cranial deformity, and any orbital- or optic-related disorders were not included in the control group.

\section{PTC group}

For this group, severe headache of unknown cause and normal MRI scans were the primary inclusion criteria; among these, we selected patients who were diagnosed with PTC (International Classification of Diseases Ninth revision Clinical Modification; 348.2 Benign Intracranial Hypertension) by a neurologist. All patients diagnosed with PTC were examined by an ophthalmologist and a neuroradiologist. Patients determined to have any ophthalmological or neuroophthalmological diseases were excluded from the study.

\section{Data sources and measurements}

All MRI were performed on the Magnetom Aera 1.5T system (Siemens Healthcare, Erlangen, Germany). Brain MRIs were performed with a 24-channel head coil. The sequences analysed consisted of 5-mm 


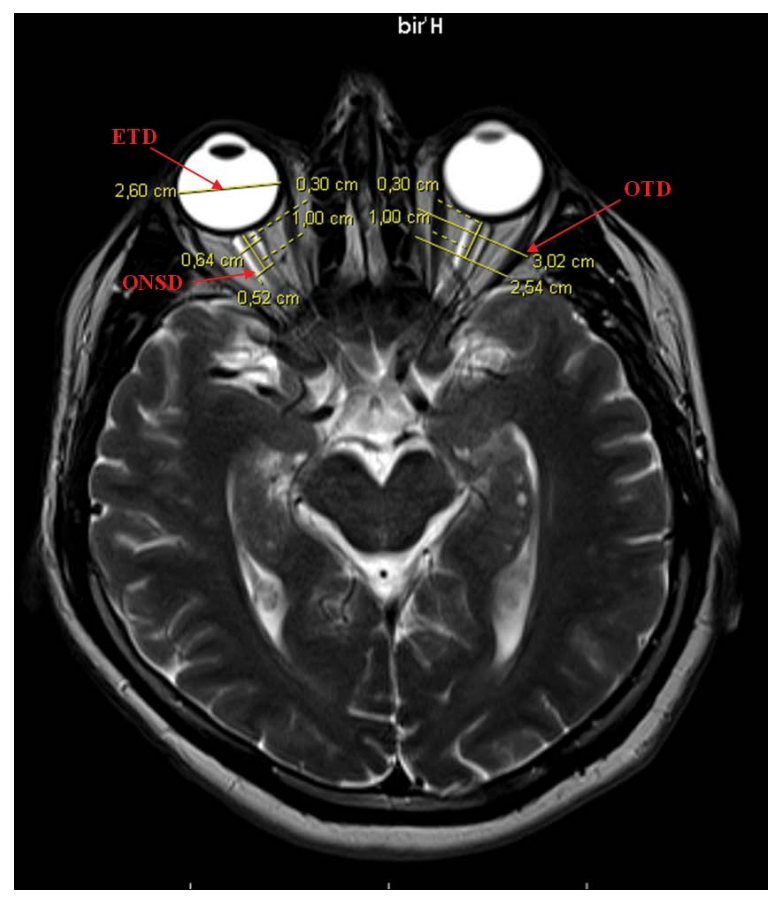

Figure 1. Axial T2W magnetic resonance imaging of the both orbit demonstrating the measurement techniques: $0 n$ the left side, orbital transverse diameter (OTD) (medial orbital wall to lateral orbital wall); on the right side, eyeball transverse diameter (ETD) (retina to retina) and optic nerve sheath diameter (ONSD) measurements $3 \mathrm{~mm}$ and $10 \mathrm{~mm}$ behind the globe were showed.

sections, and T2W axial images were used for measurements. The left and right ETD (i.e., retina to retina), OTD (i.e., medial orbital wall to lateral orbital wall), and ONSDs were measured by the computer programme on the same MRI (Fig. 1). The measurements were made digitally via PACS. Theoretical and practical orbital anatomy and measurement technique training on MRI were given to the researchers who made the measurements. MRI of patients in the control group and the PTC group were randomly mixed, so the measurements were blinded. The parts of interest of the MRI were enlarged 3-4 times for better measurement. ONSD and OTD measurements were made from $3 \mathrm{~mm}$ and $10 \mathrm{~mm}$ behind the globe (Fig. 1). The researchers took care to use the same window, brightness, and contrast when taking all measurements. Intra-evaluator and inter-evaluator consistency were analysed statistically by the intraclass correlation coefficients (ICCS) between the two evaluators. The same methodological procedures were applied by both evaluators (A.B. and T.E.) when making measurements. ICP was measured invasively in a neurosurgical intensive care unit with lumbar puncture (LP).

\section{Statistical analysis}

Statistical analysis was performed by using the Statistical Package for the Social Sciences, version 19.0 (SPSS, Chicago, Illinois). All values are presented as the mean, SD, minimum, and maximum. The distribution of the data was evaluated by the Kolmogorov-Smirnov test. A nonparametric Mann-Whitney $\mathrm{U}$ test was used to determine the differences between two groups. Correlation analysis was performed with gender and age groups. Subsequently, a receiver operating characteristic (ROC) curve was generated to determine optimal cutoff values. The results were evaluated at a $95 \%$ confidence interval, and $p<0.05$ was considered statistically significant.

\section{RESULTS}

Forty-two patients were included in the PTC group (mean age $42.49 \pm 9.15$ ), while 40 patients were included in the control group (mean age $44.03 \pm 6.81$ ). The patients in the control group were neurosurgery patients without invasive ICP results and clinical and radiological findings suggestive of normal ICP.

First, evaluator 1 measured five parameters two times. The coherence between the first and second measurements (intra-evaluator coherence) was evaluated by ICC, and the results showed an ICC value of $0.90 \leq 0.99(p<0.001)$ for correlations in all parameters. Regarding comparisons of inter-evaluator measures, a strong ICC was also seen for each variable (ICC: $0.75 \leq 0.99 ; p<0.001$ ).

\section{Comparison of the groups}

\section{Measurements made $3 \mathrm{~mm}$ posterior of the globe}

When the study group and the control group were compared, the right and left ONSDs were found to be significantly higher in the PTC group $(p \leq 0.001)$. The enlargement was bilateral, and no correlation with age was found $(p=0.68)$. Mean ONSD led to a significant prediction of PTC (area under the curve $[A \cup C]=0.988, p \leq 0.001)$. The optimal cutoff value was calculated as $4.99 \mathrm{~mm}$ with $97 \%$ sensitivity and $100 \%$ specificity. The ONSD/ETD index correlated with ICP (i.e., ICP $\uparrow$, ONSD $\uparrow$ ), and with a cutoff value of 0.21 , the sensitivity of the index was $90.5 \%$, and the specificity was $92 \%$ for measurements (AUC $=0.977$, $p \leq 0.001)$. There was no correlation between the ONSD/ETD ratio and the gender or age of the patients $(p>0.05)$. Similarly, the ONSD/OTD index correlated with ICP, and with a cutoff value of 0.17 the ONSD/ 
Table 1. Measurements made from $3 \mathrm{~mm}$ posterior of the globe

\begin{tabular}{lcccccc}
\hline \multirow{2}{*}{ Measurements } & \multicolumn{2}{c}{ Control group $(\mathbf{n}=\mathbf{4 0})$} & & \multicolumn{2}{c}{ PTC group $(\mathbf{n}=42)$} & P \\
\cline { 2 - 3 } & Mean \pm SD & Min-Max & & Mean \pm SD & Min-Max & \\
\hline ONSD & $4.444 \pm 0.397$ & $3.48-4.97$ & & $5.752 \pm 0.678$ & $4.48-7.42$ & $\mathrm{p} \leq 0.001$ \\
ONSD/ETD & $0.187 \pm 0.018$ & $0.14-0.22$ & & $0.246 \pm 0.031$ & $0.2-0.32$ & $\mathrm{p} \leq 0.001$ \\
ONSD/OTD & $0.155 \pm 0.0181$ & $0.11-0.2$ & & $0.207 \pm 0.039$ & $0.15-0.39$ & $\mathrm{p} \leq 0.001$ \\
ICP & - & - & & $28.523 \pm 7.002$ & $16-41$ & - \\
\hline
\end{tabular}

ETD — eyeball transverse diameter; ICP — intracranial pressure; ONSD — optic nerve sheath diameter; OTD — orbital transverse diameter; PTC — pseudotumour cerebri; SD — standard deviation; min — minimum; max — maximum

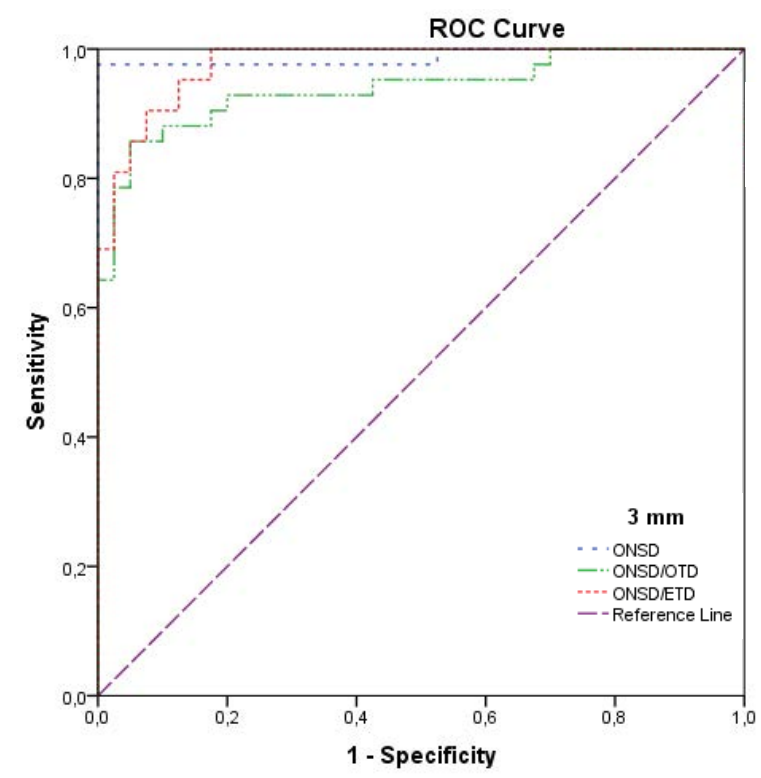

Figure 2. Receiver operating characteristic (ROC) analysis of ONSD, ONSD/ETD index and ONSD/OTD index for distance from $3 \mathrm{~mm}$ posterior of the globe; ETD — eyeball transverse diameter; ONSD optic nerve sheath diameter; OTD — orbital transverse diameter.

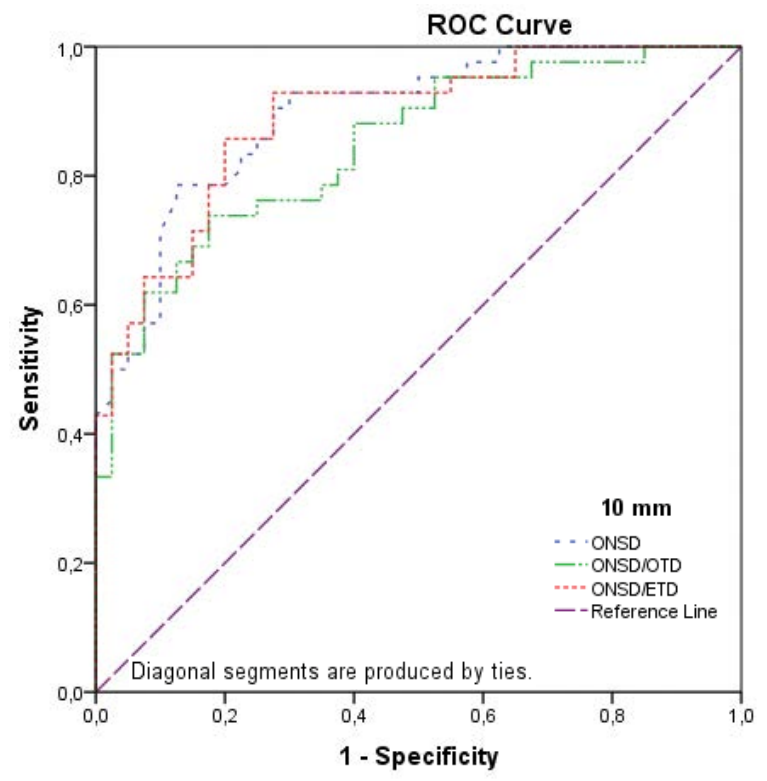

Figure 3. Receiver operating characteristic (ROC) analysis of ONSD, ONSD/ETD index and ONSD/OTD index for distance from $10 \mathrm{~mm}$ posterior of the globe; ETD — eyeball transverse diameter; ONSD — optic nerve sheath diameter; OTD — orbital transverse diameter.

Table 2. Measurements made from $10 \mathrm{~mm}$ posterior of the globe

\begin{tabular}{lcccccc}
\hline \multirow{2}{*}{ Measurements } & \multicolumn{2}{c}{ Control group $(\mathbf{n}=\mathbf{4 0})$} & & \multicolumn{2}{c}{ PTC group $(\mathbf{n}=42)$} & P \\
\cline { 2 - 3 } & Mean \pm SD & Min-Max & & Mean \pm SD & Min-Max & \\
\hline ONSD & $3.727 \pm 0.452$ & $3.05-4.77$ & & $4.782 \pm 0.797$ & $3.54-6.9$ & $\mathrm{p} \leq 0.001$ \\
ONSD/ETD & $0.156 \pm 0.019$ & $0.13-0.21$ & & $0.204 \pm 0.036$ & $0.14-0.29$ & $\mathrm{p} \leq 0.001$ \\
ONSD/0TD & $0.170 \pm 0.025$ & $0.12-0.23$ & & $0.227 \pm 0.064$ & $0.14-0.52$ & $\mathrm{p} \leq 0.001$ \\
ICP & - & - & & $28.523 \pm 7.002$ & $16-41$ & - \\
\hline
\end{tabular}

ETD — eyeball transverse diameter; ICP — intracranial pressure; ONSD — optic nerve sheath diameter; OTD — orbital transverse diameter; PTC — pseudotumour cerebri; SD — standard deviation; min — minimum; max — maximum

/OTD index had a specificity of $86 \%$ and a sensitivity of 95\% (AUC $=0.939, p \leq 0.001$ ) (Table 1, Fig. 2).

\section{Measurements made $10 \mathrm{~mm}$ posterior of the globe}

We determined the mean ONSDs for the control and PTC groups to be $4.78 \mathrm{~mm}$ and $3.72 \mathrm{~mm}$, respectively. The difference was statistically significant $(p \leq 0.001)$. The optimal cutoff value was $4.21 \mathrm{~mm}$ with $79 \%$ sensitivity and $87.5 \%$ specificity (AUC $=0.894$, $\mathrm{p} \leq 0.001)$. The sensitivity and specificity of the ONSD/ /ETD and ONSD/OTD indices were $86 \%$ and $80 \%$ and $74 \%$ and $82.5 \%$, respectively. The optimal cutoff values were calculated as 0.17 ( AUC $=0.889, p \leq 0.001$ ) and 0.19 (AUC $=0.846, p \leq 0.001)$ (Table 2, Fig. 3). 
Table 3. Measurements of the bilateral ONSDs and two indices for the control and PTC group

\begin{tabular}{|c|c|c|c|c|c|}
\hline \multicolumn{2}{|c|}{ Measurements } & \multicolumn{2}{|c|}{$\begin{array}{c}\text { Control group }(n=40) \\
\text { Mean } \pm S D\end{array}$} & \multicolumn{2}{|c|}{$\begin{array}{c}\text { PTC group }(n=42) \\
\text { Mean } \pm \text { SD }\end{array}$} \\
\hline & & Right & Left & Right & Left \\
\hline \multirow[t]{2}{*}{ ONSD } & $3 \mathrm{~mm}$ & $4.473 \pm 0.391$ & $4.416 \pm 0.415$ & $5.719 \pm 0.69$ & $5.785 \pm 0.708$ \\
\hline & $10 \mathrm{~mm}$ & $3.737 \pm 0.509$ & $3.717 \pm 0.450$ & $4.780 \pm 0.761$ & $4.783 \pm 0.868$ \\
\hline \multirow[t]{2}{*}{ ONSD/ETD } & $3 \mathrm{~mm}$ & $0.187 \pm 0.019$ & $0.186 \pm 0.019$ & $0.245 \pm 0.033$ & $0.247 \pm 0.032$ \\
\hline & $10 \mathrm{~mm}$ & $0.156 \pm 0.022$ & $0.156 \pm 0.019$ & $0.205 \pm 0.036$ & $0.204 \pm 0.038$ \\
\hline \multirow[t]{2}{*}{ ONSD/OTD } & $3 \mathrm{~mm}$ & $0.155 \pm 0.018$ & $0.155 \pm 0.019$ & $0.205 \pm 0.038$ & $0.209 \pm 0.041$ \\
\hline & $10 \mathrm{~mm}$ & $0.170 \pm 0.029$ & $0.171 \pm 0.024$ & $0.228 \pm 0.064$ & $0.226 \pm 0.066$ \\
\hline
\end{tabular}

ETD — eyeball transverse diameter; ICP — intracranial pressure; ONSD — optic nerve sheath diameter; OTD — orbital transverse diameter; PTC — pseudotumour cerebri; SD — standard deviation

In addition, the increase in ONSD was found to be statistically significant for both the right and left sides in the measurements made from both distances in the patient group (from $3 \mathrm{~mm}$ and $10 \mathrm{~mm}, \mathrm{p} \leq 0.001$ ). This result is an indirect indication of a significant increase in ICP in the patient group.

The distribution of the bilateral ONSDs and two indices of the patients for the control and PTC groups are shown in Table 3. There was no significant difference between measurements of ONSD and calculations for two indices between the right and left sides $(p>0.05)$.

\section{DISCUSSION}

One of the most accurate and simplest invasive methods for measuring ICP is LP. This indirect and imprecise procedure is still commonly used, but the invasive nature of the method, the need for a doctor to perform the procedure, technical difficulties, and unwanted complications such as bleeding and infection are risk factors for this method [23].

Because of these risk factors, researchers have sought noninvasive approaches to determine ICP. Different methods of measuring ONSD have been previously shown in many studies: in postmortem samples (direct measurement) and in patients with increased intracranial pressure by noninvasive ultrasound, $\mathrm{CT}$, or MRI $[6,12,15,16]$. It has been determined that indirect measurement of ICP using ONSD measurements on MRI is reliably associated with invasive ICP measurement $[10,14]$.

An increase in ICP appears in the majority of cranial pathologies [7]. Different thresholds have been defined to show the increase in ICP in various populations. Previous studies reported a direct relationship between increased ICP and ONSD in patients with traumatic and nontraumatic brain injuries $[3,8,14$, $22,24,26]$. Similarly, in our study, we verified recent results, showing that a linear regression model demonstrates a strong correlation between ICP and ONSD.

We can address the discussion from two sides: First, what are the results of ONSD measurements in patients with PTC and are they compatible with the literature? Second, what is the methodological comparison of the measurements we used in the study?

Optic nerve sheath diameter is calculated by various radiological methods in the literature, but as a result of those studies, the researchers did not introduce generally accepted protocols to standardise the use of the ONSD technique. Some authors reported ONSD readings taken from different distances from the globe. Therefore, there may be differences between our results and those results [11, 13-15].

In the study comparing different location points far from the eyeball for ONSD measurements, it was found that the most stable results were obtained when the diameter was measured at a distance of $10 \mathrm{~mm}$ from the globe [27]. In the present study, we measured ONSD distances from $3 \mathrm{~mm}$ and $10 \mathrm{~mm}$ posterior to the globe on MRI. We found that the ONSD measurement in patients with PTC was significantly greater than that in the control group at both distances. Moreover, in the ROC analysis to analyse the predictive value of measurements for PTC diagnosis, the diagnostic accuracy of ONSD at $3 \mathrm{~mm}$ was slightly higher than that found for ONSD at $10 \mathrm{~mm}$. We detected positive and statistically significant correlations between the LP pressure and ONSD measures for both the $3 \mathrm{~mm}(r=0.708, p \leq 0.001)$ and $10 \mathrm{~mm}(r=0.572, p \leq 0.001)$ distances. 
On the other hand, in the literature an individual approach is recommended for the application of the ONSD method. Although ONSD and LP are related in a single patient, they can differ significantly from patient to patient. While $6.6 \mathrm{~mm}$ ONSD was measured at $5 \mathrm{mmHg}$ ICP in one patient, $7 \mathrm{~mm}$ ONSD was measured at $30 \mathrm{mmHg} I \mathrm{ICP}$ in the other patient. It should be noted that ONSD measurements depend on the elasticity, extensibility, and thickness of the dura mater exhibiting individual variations $[5,29]$. Therefore, ICP assessment based on ONSD measurement in clinical examination should only be considered individually, evaluating the first and subsequent ONSD and ICP results together and making specific calculations for a particular patient.

In patients with PTC, the ONSD/ETD ratio was significantly greater than that in the control group, and it showed a positive correlation with ICP. Furthermore, their sensitivity and specificity were high for both indices calculated from $3 \mathrm{~mm}$ and $10 \mathrm{~mm}$ posterior of the globe. In the literature, only one study was performed related to ONSD/ETD and ICP in cases with hypertension. The authors found that the ONSD/ETD ratio was $0.29 \pm 0.04$ against $0.19 \pm$ \pm 0.02 in healthy adults and that the ONSD/ETD index correlated well with ICP both during the development of the pathology (i.e., ICP $\uparrow$, ONSD $\uparrow$ ) and during recovery (i.e., ICP $\downarrow$, ONSD $\downarrow$ ) [3]. As far as we know, there is no study investigating the relationship between the ONSD/OTD index and ICP in patients with PTC and other patient groups. The ONSD/ETD index correlated with ICP in patients with PTC, and its sensitivity and specificity were high. Furthermore, the ONSD/OTD index calculation $3 \mathrm{~mm}$ distance from the globe showed greater sensitivity and specificity than the index calculated $10 \mathrm{~mm}$ distance later.

The SD is a number that measures the spread of a data distribution. A high SD means that the numbers are spread out more. A low SD across studies indicates that the data points are close to the mean. ONSDs reported in the same studies have wide SDs that often overlap in the control and patient groups. To improve this situation, researchers have suggested indices such as ONS/ONSD and ONSD/ETD; these indices have insignificant SD $[6,27]$. In our study, while the SD of the ONSD measurements varied from 0.39 to 0.79 at various locations, the SDs of the ONSD/ /ETD and ONSD/OTD indices were 0.018-0.064 and $0.018-0.038$, respectively, which ensured very precise normative data.

\section{Limitations of the study}

We have two potential limitations. The first is the number of patients, and the second is the slice thickness of the MRI. Although the methodology of our study was well-founded, it consisted of a relatively small number of PTC patients. Future studies would be useful with more PTCs that could validate the results of our study. In the MRI technique, we used in our study, our slice thickness varied between 3 and $4 \mathrm{~mm}$. The use of scanners with thinner sections may be useful for more accurate analysis of the optic nerve.

\section{CONCLUSIONS}

Our study shows that MRI findings, including ONSD, ONSD/ETD and ONSD/OTD indices, are reliable neuroradiological measures to diagnose PTC in patients. In cases with high ICP, ONSD and ONSD/ETD and ONSD/OTD ratios provide readings corresponding to ICP readings in millimetres of mercury. The use of these methods in clinical practice may help provide early accurate diagnosis and early treatment, but the limitations of these methods should be taken into account.

Other imaging modalities, such as orbital MRI or MR venography, may be added to routine clinical examinations to more accurately diagnose, detail the findings and investigate new features of PTC.

\section{Conflict of interest: None declared}

\section{REFERENCES}

1. Ambika S, Arjundas D, Noronha V, et al. Clinical profile, evaluation, management and visual outcome of idiopathic intracranial hypertension in a neuro-ophthalmology clinic of a tertiary referral ophthalmic center in India. Ann Indian Acad Neurol. 2010; 13(1): 37-41, doi: 10.4103/09722327.61275, indexed in Pubmed: 20436745.

2. Baheti NN, Nair M, Thomas SV. Long-term visual outcome in idiopathic intracranial hypertension. Ann Indian Acad Neurol. 2011; 14(1): 19-22, doi: 10.4103/09722327.78044, indexed in Pubmed: 21633609.

3. Bekerman I, Sigal T, Kimiagar I, et al. The quantitative evaluation of intracranial pressure by optic nerve sheath diameter/eye diameter CT measurement. Am J Emerg Med. 2016; 34(12): 2336-2342, doi: 10.1016/j. ajem.2016.08.045, indexed in Pubmed: 27717720.

4. Caffery TS, Perret JN, Musso MW, et al. Optic nerve sheath diameter and lumbar puncture opening pressure in nontrauma patients suspected of elevated intracranial pressure. Am J Emerg Med. 2014; 32(12): 1513-1515, doi: 10.1016/j.ajem.2014.09.014, indexed in Pubmed: 25284485 .

5. Chauvet D, Carpentier A, Allain JM, et al. Histological and biomechanical study of dura mater applied to the 
technique of dura splitting decompression in Chiari type I malformation. Neurosurg Rev. 2010; 33(3): 287-295, doi: 10.1007/s10143-010-0261-x, indexed in Pubmed: 20440557.

6. Chen H, Ding GS, Zhao YC, et al. Ultrasound measurement of optic nerve diameter and optic nerve sheath diameter in healthy Chinese adults. BMC Neurol. 2015; 15: 106, doi: 10.1186/s12883-015-0361-x, indexed in Pubmed: 26148482.

7. Dunn LT. Raised intracranial pressure. J Neurol Neurosurg Psychiatry. 2002; 73(suppl 1): i23-i27, doi: 10.1136/ jnnp.73.suppl_1.i23.

8. Fraunfelder FT, Samples J, Fraunfelder FW. Possible optic nerve side effeects associated with nonsteroidal anti-inflammatory drugs. J Toxicol: Cutaneous Ocular Toxicol. 2008; 13(4): 311-316, doi: 10.3109/15569529409037531.

9. Geeraerts $T$, Launey $Y$, Martin L, et al. Ultrasonography of the optic nerve sheath may be useful for detecting raised intracranial pressure after severe brain injury. Intensive Care Med. 2007; 33(10): 1704-1711, doi: 10.1007/ s00134-007-0797-6, indexed in Pubmed: 17668184.

10. Geeraerts T, Newcombe VFJ, Coles JP, et al. Use of T2-weighted magnetic resonance imaging of the optic nerve sheath to detect raised intracranial pressure. Crit Care. 2008; 12(5): R114, doi: 10.1186/cc7006, indexed in Pubmed: 18786243.

11. Görkem SB, Doğanay S, Canpolat M, et al. MR imaging findings in children with pseudotumor cerebri and comparison with healthy controls. Childs Nerv Syst. 2015; 31(3): 373-380, doi: 10.1007/s00381-014-2579-0, indexed in Pubmed: 25358812.

12. Hassen GW, Bruck I, Donahue J, et al. Accuracy of optic nerve sheath diameter measurement by emergency physicians using bedside ultrasound. J Emerg Med. 2015; 48(4): 450-457, doi: 10.1016/j.jemermed.2014.09.060, indexed in Pubmed: 25497897.

13. Helmke $K$, Hansen HC. Fundamentals of transorbital sonographic evaluation of optic nerve sheath expansion under intracranial hypertension. I. Experimental study. Pediatr Radiol. 1996; 26(10): 701-705, doi: 10.1007/BF01383383, indexed in Pubmed: 8805599.

14. Kimberly HH, Shah S, Marill K, et al. Correlation of optic nerve sheath diameter with direct measurement of intracranial pressure. Acad Emerg Med. 2008; 15(2): 201-204, doi: 10.1111/j.1553-2712.2007.00031.x, indexed in Pubmed: 18275454.

15. Legrand A, Jeanjean $P$, Delanghe $F$, et al. Estimation of optic nerve sheath diameter on an initial brain computed tomography scan can contribute prognostic information in traumatic brain injury patients. Crit Care. 2013; 17(2): R61, doi: 10.1186/cc12589, indexed in Pubmed: 23536993.

16. Liu D, Kahn M. Measurement and relationship of subarachnoid pressure of the optic nerve to intracranial pressures in fresh cadavers. Am J Ophthalmol. 1993; 116(5): 548-556, doi: 10.1016/s0002-9394(14)73195-2, indexed in Pubmed: 8238213.

17. Lochner P, Mader C, Nardone R, et al. Sonography of the optic nerve sheath beyond the hyperacute stage of intracerebral hemorrhage. J Ultrasound. 2014; 17(3): 225-228, doi: 10.1007/s40477-014-0069-6, indexed in Pubmed: 25177397.
18. McMahon CJ, McDermott P, Horsfall D, et al. The reproducibility of transcranial Doppler middle cerebral artery velocity measurements: implications for clinical practice. Br J Neurosurg. 2007; 21(1): 21-27, doi: 10.1080/02688690701210539, indexed in Pubmed: 17453770 .

19. Pal A, Sengupta P, Biswas D, et al. Pattern of idiopathic intracranial hypertension in Indian population. Ann Indian Acad Neurol. 2019; 22(1): 47-51, doi: 10.4103/aian. AIAN 116 18, indexed in Pubmed: 30692759.

20. Pearce JMS. From pseudotumour cerebri to idiopathic intracranial hypertension. Pract Neurol. 2009; 9(6): 353-356, doi: 10.1136/jnnp.2009.194837, indexed in Pubmed: 19923117.

21. Raboel PH, Bartek J, Andresen M, et al. Intracranial pressure monitoring: invasive versus non-invasive methods: a review. Crit Care Res Pract. 2012; 2012: 950393, doi: 10.1155/2012/950393, indexed in Pubmed: 22720148.

22. Raffiz M, Abdullah JM. Optic nerve sheath diameter measurement: a means of detecting raised ICP in adult traumatic and non-traumatic neurosurgical patients. Am J Emerg Med. 2017; 35(1): 150-153, doi: 10.1016/j. ajem.2016.09.044, indexed in Pubmed: 27852525.

23. Rickert K, Sinson G. Intracranial pressure monitoring. Oper Tech Gen Surg. 2003; 5(3): 170-175, doi: 10.1016/ s1524-153x(03)70010-4.

24. Sekhon MS, Griesdale DE, Robba C, et al. Optic nerve sheath diameter on computed tomography is correlated with simultaneously measured intracranial pressure in patients with severe traumatic brain injury. Intensive Care Med. 2014; 40(9): 1267-1274, doi: 10.1007/s00134-0143392-7, indexed in Pubmed: 25034476.

25. Spennato P, Ruggiero C, Parlato RS, et al. Pseudotumor cerebri. Childs Nerv Syst. 2011; 27(2): 215-235, doi: 10.1007/ s00381-010-1268-x, indexed in Pubmed: 20721668.

26. Tayal VS, Neulander M, Norton HJ, et al. Emergency department sonographic measurement of optic nerve sheath diameter to detect findings of increased intracranial pressure in adult head injury patients. Ann Emerg Med. 2007; 49(4): 508-514, doi: 10.1016/j.annemergmed.2006.06.040, indexed in Pubmed: 16997419.

27. Vaiman M, Gottlieb P, Bekerman I. Quantitative relations between the eyeball, the optic nerve, and the optic canal important for intracranial pressure monitoring. Head Face Med. 2014; 10: 32, doi: 10.1186/1746-160X-10-32, indexed in Pubmed: 25130267.

28. Vaiman M, Sigal T, Kimiagar I, et al. Noninvasive assessment of the intracranial pressure in non-traumatic intracranial hemorrhage. J Clin Neurosci. 2016; 34: 177-181, doi: 10.1016/j.jocn.2016.06.008, indexed in Pubmed: 27612672.

29. Van Noort R, Martin TR, Black MM, et al. The mechanical properties of human dura mater and the effects of storage media. Clin Phys Physiol Meas. 1981; 2(3): 197-203, doi: 10.1088/0143-0815/2/3/003, indexed in Pubmed: 7338023.

30. Zaidi SJ, Yamamoto LG. Optic nerve sheath diameter measurements by CT scan in ventriculoperitoneal shunt obstruction. Hawaii J Med Public Health. 2014; 73(8): 251-255, indexed in Pubmed: 25157326. 